ARTICLE

\title{
Boosting photoelectrochemical efficiency by near-infrared-active lattice-matched morphological heterojunctions
}

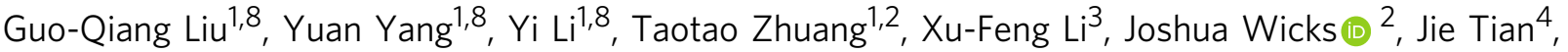 \\ Min-Rui Gao (1) ${ }^{1 \times}$, Jin-Lan Peng ${ }^{5}$, Huan-Xin Ju${ }^{6}$, Liang Wu${ }^{1}$, Yun-Xiang Pan ${ }^{7}$, Lu-An Shi ${ }^{1}$, Haiming Zhu (1) ${ }^{3}$,

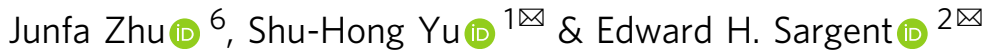

Photoelectrochemical catalysis is an attractive way to provide direct hydrogen production from solar energy. However, solar conversion efficiencies are hindered by the fact that light harvesting has so far been of limited efficiency in the near-infrared region as compared to that in the visible and ultraviolet regions. Here we introduce near-infrared-active photoanodes that feature lattice-matched morphological hetero-nanostructures, a strategy that improves energy conversion efficiency by increasing light-harvesting spectral range and charge separation efficiency simultaneously. Specifically, we demonstrate a near-infraredactive morphological heterojunction comprised of BiSeTe ternary alloy nanotubes and ultrathin nanosheets. The heterojunction's hierarchical nanostructure separates charges at the lattice-matched interface of the two morphological components, preventing further carrier recombination. As a result, the photoanodes achieve an incident photon-to-current conversion efficiency of $36 \%$ at $800 \mathrm{~nm}$ in an electrolyte solution containing hole scavengers without a co-catalyst.

\footnotetext{
${ }^{1}$ Division of Nanomaterials \& Chemistry, Hefei National Laboratory for Physical Sciences at the Microscale, Institute of Energy, Hefei Comprehensive National Science Center, CAS Center for Excellence in Nanoscience, Department of Chemistry, Institute of Biomimetic Materials \& Chemistry, Anhui Engineering Laboratory of Biomimetic Materials, University of Science and Technology of China, Hefei, China. ${ }^{2}$ Department of Electrical and Computer Engineering, University of Toronto, 35 St George Street, Toronto, ON, Canada. ${ }^{3}$ Department of Chemistry, Zhejiang University, Hangzhou, Zhejiang, China. ${ }^{4}$ Engineering and Materials Science Experiment Center, University of Science and Technology of China, Hefei, Anhui, China. ${ }^{5}$ Center for Micro- and Nanoscale Research and Fabrication, University of Science and Technology of China, Hefei, Anhui, China. ${ }^{6}$ National Synchrotron Radiation Laboratory, University of Science and Technology of China, Hefei, China. ${ }^{7}$ School of Chemistry and Chemical Engineering, Hefei University of Technology, Hefei, Anhui, China. ${ }^{8}$ These authors contributed equally: Guo-Qiang Liu, Yuan Yang, Yi Li.『email: mgao@ustc.edu.cn; shyu@ustc.edu.cn; ted.sargent@utoronto.ca
} 
T he direct conversion of solar energy into chemical fuels offers a means to store renewable energy ${ }^{1-8}$. However, the practical application of photoelectrochemical (PEC) hydrogen production is impeded today by its low energy conversion efficiency ${ }^{9-13}$.

The primary obstacle limiting hole-involved photoelectrocatalytic reactions is the low concentration of photoholes $\left(C_{h+}\right)$ that reach the surface of catalysts ${ }^{14-16}$. Improving the number of available photons to increase $C_{h+}$ represents an important avenue to achieving high-efficiency photoanodes.

A wide range of semiconductors ${ }^{17-21}$ have shown application as photoanode materials; however, their large bandgaps limit light absorption to the ultraviolet (UV, below $400 \mathrm{~nm}$ ) and visible (vis, 400 to $700 \mathrm{~nm}$ ) wavelengths. Extending optical absorption into the infrared region (IR, above $700 \mathrm{~nm}$ ) enables further utilization of the remaining $50 \%$ of solar photon fluence and will promote these devices in toward the Shockley-Queisser (SQ) efficiency limit ${ }^{17,22}$. Upconverting phosphors ${ }^{23,24}$ and metal plasmonic nanostructures $^{25}$ have previously been integrated into nearinfrared (NIR) photoelectrodes (Supplementary Table 1). Unfortunately, the low efficiency of upconversion luminescence and the short sub-picosecond lifetime of hot charge carriers lead to impractical photocurrent densities on the order of microamperes per square centimeter ${ }^{26-28}$.

Narrow bandgap (NBG) semiconductors ${ }^{18}$ that exhibit a wide NIR absorption range, a large absorption cross section, and longlived charge carriers, offer a promising avenue toward NIR-active photoelectrodes that utilize band-edge carriers. However, electron-phonon interactions and non-radiative recombination at defects have, until now, resulted in short-lived photoexcited carriers in these NBG semiconductors, a fact that prevents the needed surface redox reactions from proceeding efficiently ${ }^{27}$. Consequently, NIR absorber-based photoanodes have been limited to an incident photon-to-current conversion efficiency (IPCE) below 3\% ${ }^{18,29-31}$.

With these considerations in mind, we focused on the development of NIR-active morphological heterostructures (MHs) with lattice-matched interfaces as a means to enhance the energy conversion efficiency of NBG semiconductors. Lattice-matched morphological heterojunctions reduce carrier-trapping interfacial defects that generally exist at interfaces of two distinct semiconductors with large lattice mismatch ${ }^{32,33}$. When the morphological heterojunction is selected for a broadband absorption spectral range, and when it provides a low recombination rate, it offers avenues to increased energy conversion efficiencies.

Here, we report an efficient NIR-active photoanode by engineering a BiSeTe ternary alloy in the form of lattice-matched morphological heterojunctions (BST-MHs). We grow epitaxially thin nanosheets onto nanotubes that absorb NIR radiation up to $1100 \mathrm{~nm}$. We verify the existence of a lattice-matched type-II heterojunction and the ultrafast charge transfer process responsible for enhancing electron-hole separation. The resulting photoanodes exhibit an IPCE that reaches $36 \%$ at $800 \mathrm{~nm}$ in an electrolyte solution containing hole scavengers without a cocatalyst.

\section{Results}

BST-MH synthesis and characterization. Bismuth chalcogenides $\left(\mathrm{Bi}_{2} \mathrm{Te}_{3}\right.$ and $\left.\mathrm{Bi}_{2} \mathrm{Se}_{3}\right)$ with bulk bandgaps of $0.15-0.35 \mathrm{eV}$ and optical absorption coefficients, $\alpha$, on the order of $10^{5} \mathrm{~cm}^{-1}$ hold potential as NIR absorption materials ${ }^{34-36}$. However, such NBGs usually lead to fast electron-hole recombination under solar irradiation, thus limiting the solar conversion efficiency ${ }^{27}$.

Fortunately, the quantum confinement of few-layer bismuth chalcogenides with thicknesses below their exciton Bohr radii
(52.5 $\mathrm{nm}$ for $\mathrm{Bi}_{2} \mathrm{Se}_{3}$ ) enables them layer-dependent carrier mobilities (on the order of $10^{3}$ to $10^{4} \mathrm{~cm}^{2} \mathrm{~V}^{-1} \mathrm{~s}^{-1}$ ) and tailored bandgaps ${ }^{37,38}$. We hypothesized that building lattice-matched type-II morphological heterojunctions based on quantumconfined ternary BiSeTe semiconductor materials with suitable bandgaps could enable engineering photoanodes toward more efficient NIR-driven solar energy conversion.

We designed BiSeTe morphological heterojunctions (BSTMHs) using a strategy that combines chemical transformation with epitaxial growth. The BST-MHs-constructed in the form that few-layer nanosheets epitaxially grow on nanotubes-were chemically transformed from templates of $\mathrm{Te}_{\mathrm{x}} \mathrm{Se}_{\mathrm{y}} @ \mathrm{Se}$ core-shell nanowires (Supplementary Fig. 1, see Methods for further synthetic details) ${ }^{39,40}$. We examined the BST-MHs using lowmagnification transmission electron microscopy (TEM, Fig. 1a): these revealed hierarchical nanostructures with ultrathin nanosheets grown around the nanotubes. We further confirmed the ultrathin nature of the nanosheets via atomic force microscopy (AFM, Fig. 1b). The hierarchical nanostructure is expected to enable both rapid carrier transport to improve the charge separation, and also a high surface-to-volume ratio to facilitate redox reactions.

The peaks in the powder X-ray diffraction (XRD) pattern of BST-MHs (Supplementary Fig. 2a) were indexed to the rhombohedra-phase $\mathrm{Bi}_{2} \mathrm{Se}_{3}$ (JCPDS card No. 33-0214, space group of R-3m) with an overall shift to lower $2 \theta$ values, a finding we attribute to the incorporation of tellerium ${ }^{39}$. Elemental mapping images of the BST-MHs (Fig. 1c) and energy dispersive spectra (EDS) (Supplementary Fig. 2b-d) revealed that the Bi and Se elements are homogeneously distributed in the nanotubes and nanosheets; while $\mathrm{Te}$ is mainly distributed in the nanotubes, consistent with the core-shell structure of the $\mathrm{Te}_{\mathrm{x}} \mathrm{Se}_{\mathrm{y}} @ \mathrm{Se}$ nanowire templates.

High-resolution transmission electron microscopy (HRTEM) images of the BiSeTe nanotubes and nanosheets (Supplementary Figs. 3 and 4) show lattice spacings of 0.207 and $0.305 \mathrm{~nm}$, which we index to the (110) and (015) planes of the rhombohedral BiSeTe ternary compounds, respectively. Each corresponding fast Fourier transform (FFT) image exhibits the diffraction pattern associated with rhombohedral BiSeTe, matching well with XRD patterns.

We further carried out HRTEM to investigate lattice registration at the nanotube/nanosheet interface. Considering that ultrathin nanosheets randomly grow around the nanotubes in a hierarchical manner, we cross-sectioned the unidirectionally aligned nanowires to observe them along different directions. HRTEM images on the nanotubes, nanosheets, and tube/sheet interfaces show lattice spacing of $0.305 \mathrm{~nm}$, which we index to the (015) plane of BiSeTe ternary compounds (Fig. 1d-o). FFTs obtained from these HRTEM images indicate a single diffraction pattern assigned to the [100] crystal zone axis of BiSeTe. Similar results can also be found in Supplementary Figs. 5-7. We propose that the lattice-matched epitaxial growth reduces detrimental defects at the interface, which would contribute to efficient charge separation.

Energy band alignment of BST-MHs. Reducing the thickness of the BiSeTe ternary alloy down to a quantum-confined size is expected to change the energy band structure and corresponding optical properties, and avoid strong electron-phonon scattering ${ }^{41-43}$. To investigate the band alignment of BST-MHs in detail, we tuned the shape of the ternary alloy BiSeTe material to follow only a nanotube (BST-NT) or a nanosheet (BST-NS) structure while retaining the same components and crystal structures for comparison (Supplementary Fig. 8). HRTEM 
a

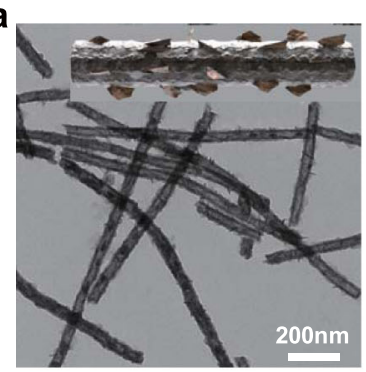

b

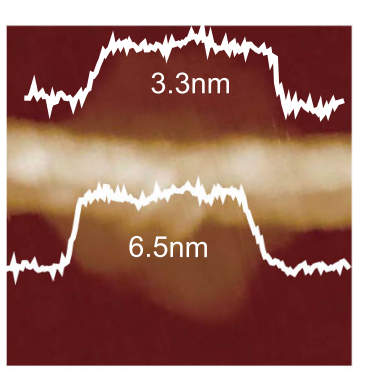

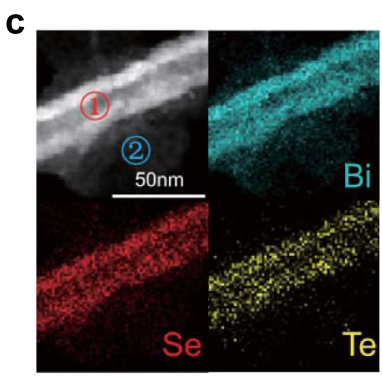

d
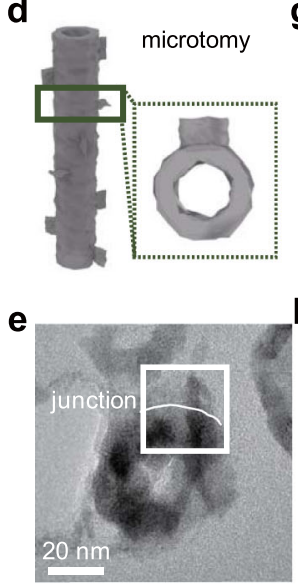

f

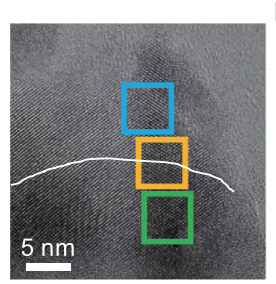

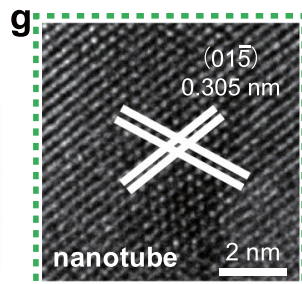

h

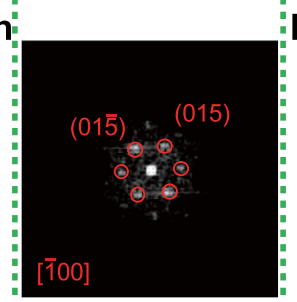

i

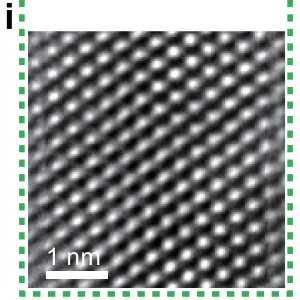

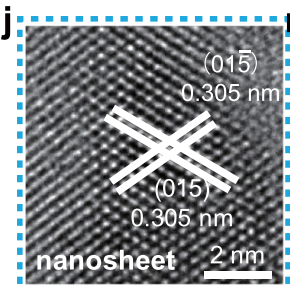
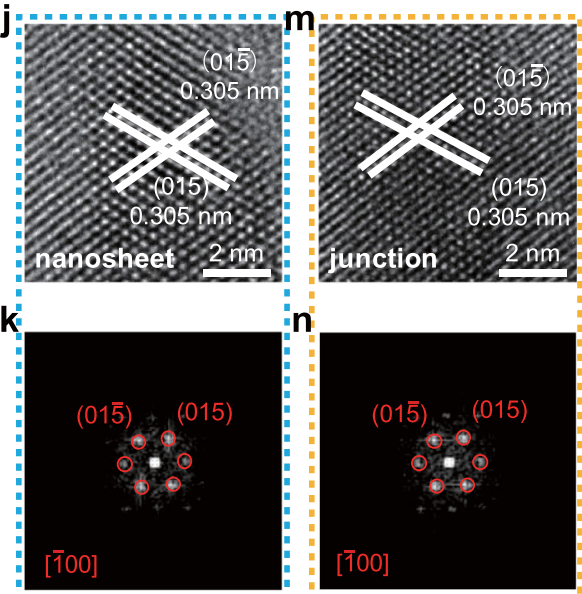

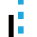

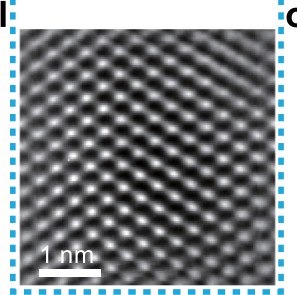

0

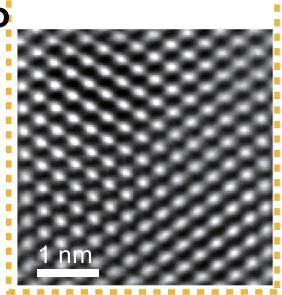

Fig. 1 Morphological and compositional characterization of BST-MHs. a TEM image of BST-MHs. Inset is a schematic geometric model. b AFM image of BST-MHs. c EDS mapping images of BST-MHs. $\mathbf{d}$ Schematic of the sliced BST-MHs. e, $\mathbf{f}$ TEM image and HRTEM image of the junction. $\mathbf{g}-\mathbf{i}$ HRTEM image, FFT, and the inverse FFT of the nanotube in the green domain of (f). j-I HRTEM image, FFT, and the inverse FFT of the nanosheet in the blue domain of (f). $\mathbf{m}-\mathbf{o}$ HRTEM image, FFT, and the inverse FFT of junction in the yellow domain of (f).

images, EDS spectra (Supplementary Fig. 9), and XPS spectra (Supplementary Fig. 10) indicated that the plain BST-NTs and BST-NSs have the crystal orientations, grain boundaries, and elemental compositions of their counterparts in BST-MHs.

To verify their optical bandgaps $\left(E_{\mathrm{g}}\right)$, we transformed the UVvis diffuse reflectance spectra into a Tauc plot (Fig. 2a). The optical bandgaps of BST-MHs, BST-NTs, and BST-NSs are 0.99, 1.00 , and $1.15 \mathrm{eV}$, respectively. In contrast to the bandgaps of bulk $\mathrm{Bi}_{2} \mathrm{Se}_{3}(0.35 \mathrm{eV})$ and bulk $\mathrm{Bi}_{2} \mathrm{Te}_{3}(0.15 \mathrm{eV})$, the BST-MH samples show larger optical bandgaps, a finding we assign to the quantum confinement effect $37,39,43-45$. We further characterized their band structures by ultraviolet photoelectron spectroscopy (UPS). Figure $2 \mathrm{~b}$ shows the relative electron binding energies between the valence band maximum and Fermi level $\left(E_{\mathrm{VBM}}-E_{\mathrm{f}}\right)$, which are $-0.77,-0.77$, and $-1.04 \mathrm{eV}$, for BST-MHs, BST-NTs, and BST-NSs. Meanwhile, we calculated, using the equation $\Phi=$ $h v-E_{\text {onset, }}$, that the work functions $(\Phi$, the relative energy between the Fermi level and the vacuum level) of BST-MHs, BSTNTs, and BST-NSs are $4.23,4.00$, and $4.50 \mathrm{eV}$, respectively (Fig. 2c).

Considering the relationship between the $E_{\mathrm{g}}, E_{\mathrm{VBM}}-E_{\mathrm{f}}$, and $\Phi$, we, therefore, present the energy band diagrams before and after alignment (Fig. 2d). The $E_{\mathrm{f}}$ difference between plain BST-NTs and BST-NSs drives electrons in BST-NTs to flow into BST-NSs after the formation of heterojunctions, resulting in Fermi level alignment at $4.23 \mathrm{eV}$. In this way, the type-II band structure formed in the BST-MHs facilitates charge transfer and separation. Under solar irradiation, photoexcited electrons in BST-NTs flow into BST-NSs, while photoexcited holes can transfer from BSTNSs into BST-NTs.

To verify the proposed band alignment and charge transfer mechanism in the BST-MHs, we further carried out femtosecond transient absorption (TA) spectroscopy on BST-NTs, BST-NSs, and BST-MHs. After photoexcitation, BST-NTs and BST-NSs instantaneously show bleach peaks centered at $\sim 645$ and $\sim 590$ $\mathrm{nm}$, respectively (Supplementary Fig. 11a, b). We attribute the bleach mainly to the state filling of conduction band edges, given the much smaller effective mass of an electron than a hole in these materials. Thus, these bleach kinetics (Fig. 2e) inform the electron population ${ }^{38,43}$. We extracted a half-lifetime of $\sim 7$ and $\sim 2$ ps from TA kinetics of BST-NTs and BST-NSs (Supplementary Table 2).

In BST-MHs, the bleach peak from the NTs at $\sim 645 \mathrm{~nm}$ appears instantaneously after photoexcitation, but decays quickly within $\sim 0.4 \mathrm{ps}$. This process is accompanied by the rising bleach peak at $\sim 590 \mathrm{~nm}$ associated with the NSs in the same timescale (Supplementary Fig. 11c), indicating an ultrafast electron transfer process from the NT domain to the NS domain in BST-MHs 
a

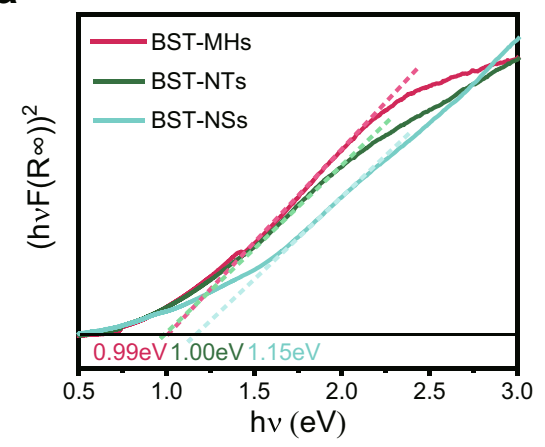

d

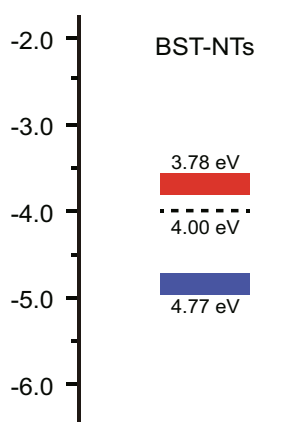

e

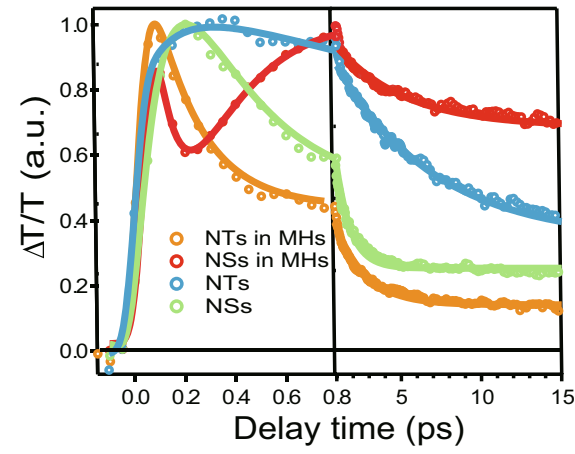

b

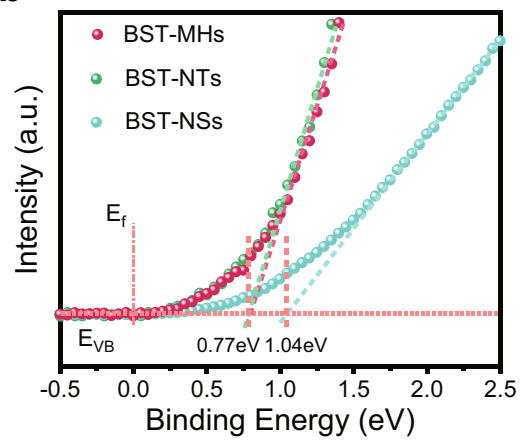

C

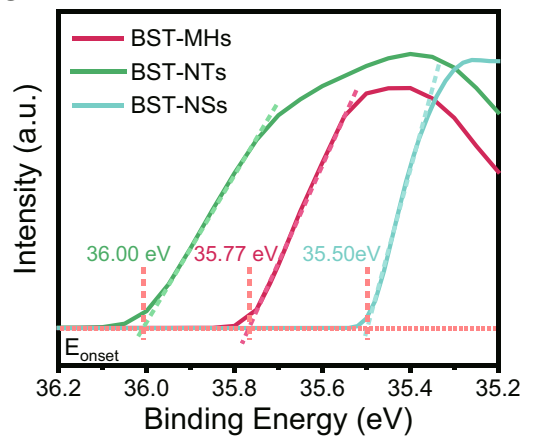

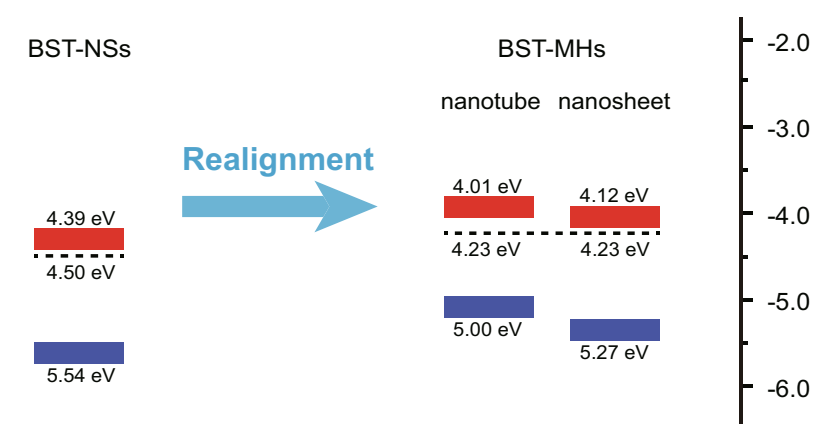

f

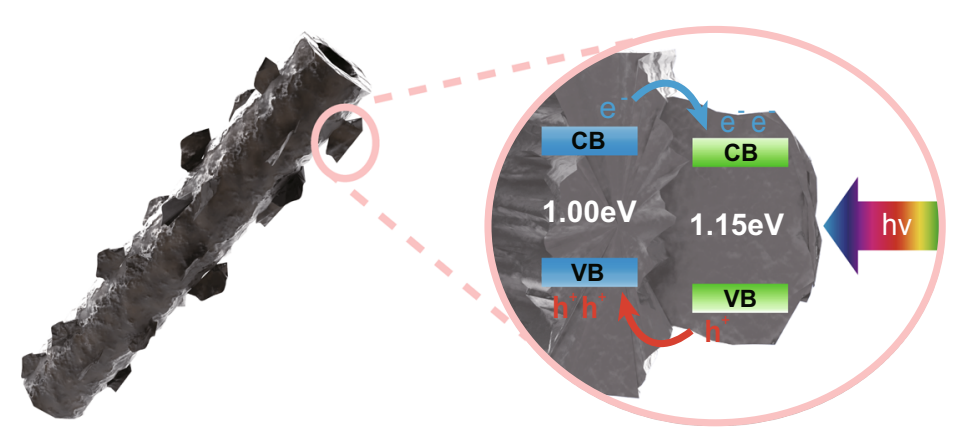

Fig. 2 Spectroscopic characterization and schematic energy band diagrams. a Tauc plots of BST-MHs, BST-NTs, and BST-NSs derived from UV-Vis diffuse reflectance spectra. b UPS spectra of BST-NTs, BST-NSs, and BST-MHs, showing their relative energies between valence band maximum and Fermi level. c Onset level $\left(E_{\text {onset }}\right)$ of the UPS spectra for BST-MHs, BST-NTs, and BST-NSs, respectively. d Energy band diagrams of BST-NTs, BST-NSs, and BSTMHs. e Corresponding TA kinetics at $B_{1}$ and $B_{2}$ of BST-NTs, BST-NSs, and BST-MHs. The circles are experimental data and the solid lines are their exponential fits. $\mathbf{f}$ Schematic of charge-separation processes in BST-MHs under solar irradiation.

driven by the type-II band alignment (Fig. 2f). The resulting electrons in the NS domain of BST-MHs have a half-lifetime of $\sim 80$ ps (Supplementary Fig. 11d), which is more than one order of magnitude longer than that in BST-NTs and BST-NSs. The much longer electron lifetime in MHs is attributed to spatial electronhole separation in such type-II morphological heterojunctions.

Photoelectrochemical hydrogen production performance. We implemented the $\mathrm{PEC} \mathrm{H}_{2}$ production half-reaction to test NIR-MHs for promise in performance. We prepared thickness-controlled photoanodes and compared their PEC performance using $\mathrm{Na}_{2} \mathrm{SO}_{3} /$ $\mathrm{Na}_{2} \mathrm{~S}$ as hole scavengers (Supplementary Figs. 12-14) ${ }^{46-48}$. The optimal thicknesses for BST-MHs, NTs, NSs, and NTs+NSs are determined to be 3, 2, 3, and 2 layers, respectively (Supplementary Fig. 15a-d).
As shown in Fig. 3a, the anodic photocurrent density of BSTMHs sharply increased at potentials above $0.5 \mathrm{~V}_{\mathrm{RHE}}$, reaching $3.7 \mathrm{~mA} \mathrm{~cm}^{-2}$ at $0.75 \mathrm{~V}_{\mathrm{RHE}}$. In stark contrast, photocurrent densities for BST-NTs and BST-NSs were 1.98 and $0.96 \mathrm{~mA} \mathrm{~cm}^{-2}$ at $0.75 \mathrm{~V}_{\mathrm{RHE}}$. The BST-MHs achieve a twofold higher photocurrent density compared with the best previous reports (Supplementary Table 3). Compared to BST-NT and BST-NS photoanodes, BST-NT + NS photoanodes show higher photocurrent due to the hierarchical structure formed by a simple stacking of nanosheets and nanotubes, which improves the absorption capacity of the photoelectrode by light scattering (Supplementary Fig. 12) ${ }^{5}$. However, the BST-MHs showed a larger photocurrent than that of the individual BST-NTs, BSTNSs, and the BST-NT + NSs. We attribute the enhanced performance of the BST-MHs to lattice-matched type-II morphological heterojunctions, which are conducive to the separation 
a

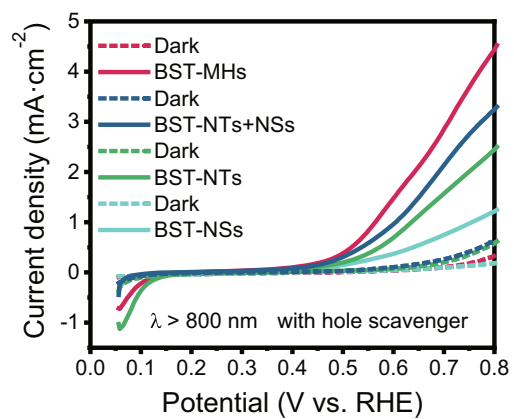

d

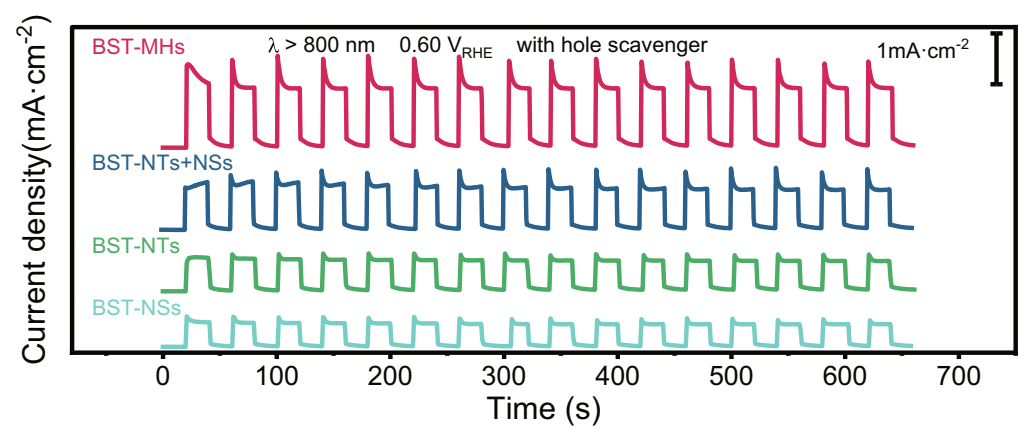

C

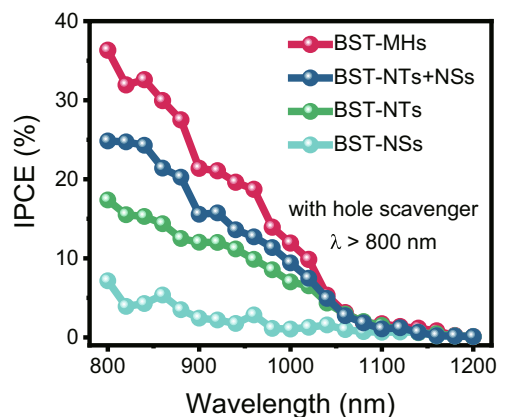

e
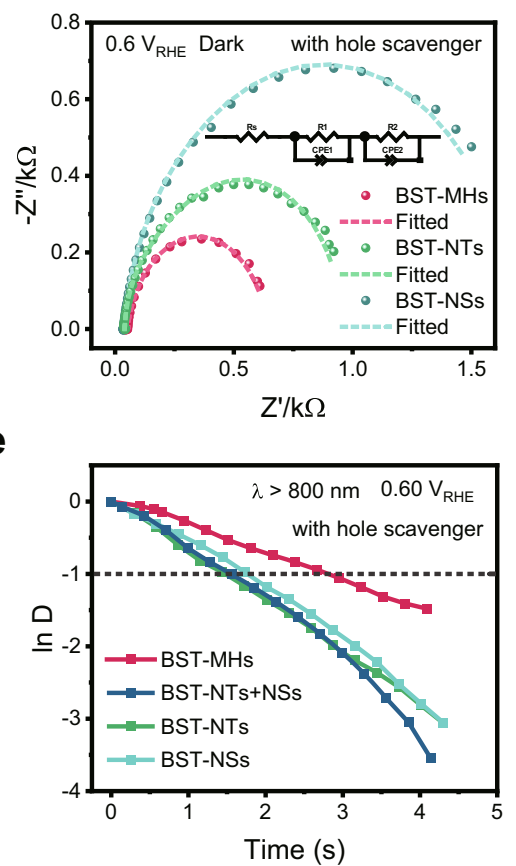

f

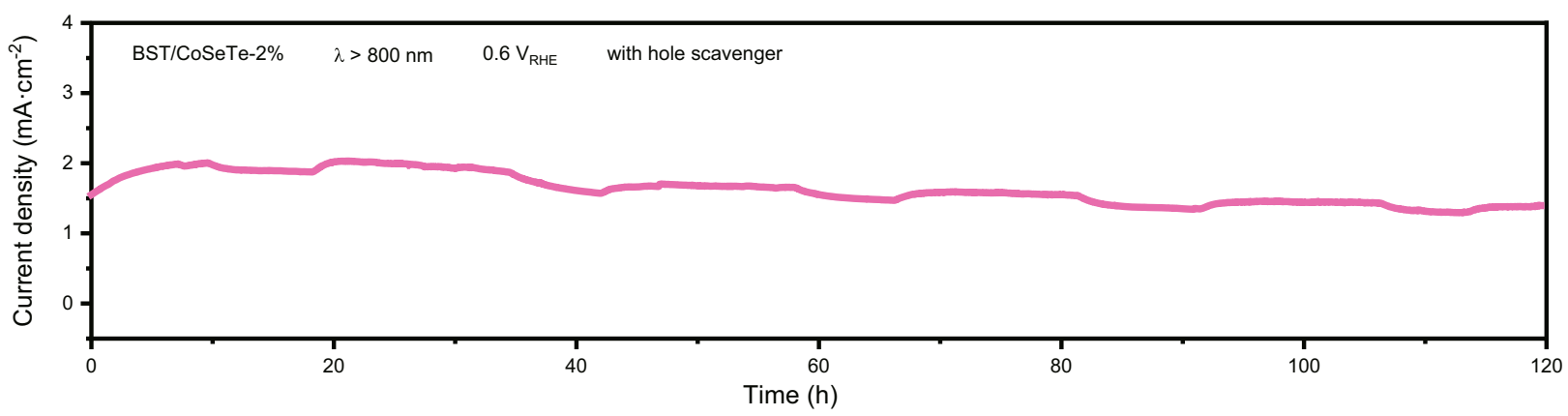

Fig. 3 PEC performance. a, b Current-potential curves (a), IPCEs (b) of the BST-MH, BST-NT, BST-NS, and BST-NT + BST-NS photoanodes under NIR light irradiation $\left(\lambda>800 \mathrm{~nm}, 100 \mathrm{~mW} \mathrm{~cm}^{-2}\right.$ ). c Electrochemical impedance spectra measured for different photoanodes with hole scavengers, $Z$ " and $Z$ " are the real and imaginary parts of impedance. The inset shows the equivalent circuit. d, e Transient current-time curves (d) and normalized plots of photocurrent density-time dependence (e) of BST-MH, BST-NT, BST-NS, and BST-NT + BST-NS photoanodes at $0.60 \mathrm{~V}_{\text {RHE }}$ under NIR light irradiation $(\lambda>800 \mathrm{~nm}$, $\left.100 \mathrm{~mW} \mathrm{~cm}^{-2}\right)$. f Stability test of the BST/CoSeTe-2\% photoanode at $0.60 \mathrm{~V}_{\mathrm{RHE}}$ under NIR light irradiation $\left(\lambda>800 \mathrm{~nm}, 100 \mathrm{~mW} \mathrm{~cm}^{-2}\right)$. All experiments were measured in an electrolyte solution containing $0.08 \mathrm{~mol} \mathrm{~L}^{-1} \mathrm{Na}_{2} \mathrm{~S}$ and $0.50 \mathrm{~mol} \mathrm{~L}^{-1} \mathrm{Na}_{2} \mathrm{SO}_{3}$ as hole scavengers $(\mathrm{pH}=12.9$ ).

and transport of charge carriers. Figure $3 \mathrm{~b}$ shows that the IPCE of BST-MHs is higher than that of BST-NTs, BST-NSs, and the mixture in the range of $800-1100 \mathrm{~nm}$. The IPCE of the BST-MH photoanode reached $36 \%$ at $800 \mathrm{~nm}$ in an electrolyte solution containing hole scavengers with an applied potential of $0.6 \mathrm{~V}_{\mathrm{RHE}}$, demonstrating a threefold enhancement compared to the existing record $^{18}$. In addition, the IPCE values of these photoanodes approached $0 \%$ at $1200 \mathrm{~nm}$, in accordance with their optical bandgaps. The high photocurrent density and IPCE can be attributed to charge separation with long-lived charge carriers driven by type-II band alignment, consistent with TA analysis and electrochemical impedance spectra (Fig. $3 \mathrm{c}$ and Supplementary Table 4). These results support the view that NIR-MHs composed of an NBG semiconductor serves as a NIR-active photoanode material.

The transient photocurrent response of the BST-MH photoanode was acquired at a constant potential of $0.60 \mathrm{~V}_{\mathrm{RHE}}$ with a light chopper under NIR light (Fig. 3d). Results show that the stable photocurrent density of BST-MHs $\left(1.1 \mathrm{~mA} \mathrm{~cm}^{-2}\right)$ is significantly enhanced compared with other photoanodes. We further analyzed the photocurrent decay at $0.6 \mathrm{~V}_{\mathrm{RHE}}$. In contrast to other photoanodes investigated in this work, the transient decay time, $\tau$, of the BST-MH photoanode is the longest, indicating that the lattice-matched $\mathrm{MHs}$ suppress carrier recombination and accelerate carrier migration (Fig. 3e). The Mott-Schottky plots reveal that the flat-band potential of the ntype BST-MHs is $0.425 \mathrm{~V}_{\mathrm{RHE}}$. We estimated the carrier concentration in the BST-MHs using the double-layer capacitance ${ }^{49}$, which was on the order of $10^{20} \mathrm{~cm}^{-3}$ (Supplementary Fig. 15e). We also measured its open-circuit photovoltage under different solar sources to estimate the attainable photovoltage under PEC operating conditions (Supplementary Fig. 15f). In addition, to achieve unassisted solar water splitting, we aim to build the next generation electrode structures to increase the photovoltage. These electrodes can be coupled with high-efficiency tandem PV-PEC cells to achieve full utilization of the solar spectrum, thus improving the energy conversion efficiency of the device ${ }^{6,7}$.

To mitigate the spike in transient current and photocorrosion of the photoanode-a consequence of the inefficient hole- 
involved sulfite oxidation reaction-we introduced a cobalt-based cocatalyst (CoSeTe) to the BST-MHs (Supplementary Figs. 1618). We tested the transient photocurrent response and the stability of the photoanode for PEC hydrogen production in electrolytes containing $\mathrm{Na}_{2} \mathrm{~S}$ and $\mathrm{Na}_{2} \mathrm{SO}_{3}$ (Fig. 3f and Supplementary Figs. 19, 20). Under NIR light, the transient decay time $\tau$ of the BST/CoSeTe-2\% photoanode is extended threefold, and the photoanode remained stable during a $120 \mathrm{~h}$ experiment. In contrast to plain BST-MHs, the BST/CoSeTe photoanode maintained its morphology after $120 \mathrm{~h}$ (Supplementary Fig. 20d, e). This demonstrates that the separation and consumption of carriers in the photoanode is accelerated after loading the cocatalyst, avoiding photocorrosion, and thereby ensuring its stability.

To challenge further the efficiency of carrier separation and application-relevance of MHs, we conducted PEC water splitting under NIR light. As shown in Supplementary Fig. 21, the lower onset potential and higher photocurrent revealed that the latticematched type-II MHs efficiently enhanced the separation and transport of photogenerated carriers. After loading a cocatalyst to accelerate water oxidation kinetics, the BST/CoSeTe- $2 \%$ photoanode showed the lowest onset potential and highest photocurrent. The BST/CoSeTe-2\% photoanode showed improved stability compared to the BST-MH photoanode. We attribute the moderate photocurrents to the fact that a more suitable cocatalyst to promote the water oxidation reaction has yet to be found. With the identification of suitable cocatalysts as a clear next step, these BiSeTe-based semiconductors provide a route to more efficient and stable PEC water splitting.

In addition, we also tested the PEC performance of the BSTMH photoanode under visible light illumination $(\lambda>420 \mathrm{~nm})$, as displayed in Supplementary Fig. 22. A significant quantity of $\mathrm{H}_{2}$ bubbles was observed on the Pt counter electrode (Supplementary movie 1). The anodic photocurrent density of BST-MHs reached $7.1 \mathrm{~mA} \mathrm{~cm}^{-2}$ at $0.75 \mathrm{~V}_{\mathrm{RHE}}$. Since sulfite oxidation is thermodynamically and kinetically more facile, BST-MH photoanodes have a lower observed overpotential, which results in the low onset potential $\left(0.2 \mathrm{~V}_{\mathrm{RHE}}\right)$ of the anode photocurrent ${ }^{12}$. The IPCE of the BST-MH photoanode reached $73 \%$ at $400 \mathrm{~nm}$ in an electrolyte solution containing hole scavengers with an applied potential of $0.6 \mathrm{~V}_{\mathrm{RHE}}$.

Further to prove the advantage of MHs, we grew two types of lattice-mismatched hetero-nanostructures (BST/ $\mathrm{Bi}_{2} \mathrm{~S}_{3}$ and BST/ $\mathrm{MoS}_{2}$ ). These had a significantly higher density of interface defects due to the larger difference in lattice spacing and crystalline orientation at the interface (Supplementary Figs. 2328 , see Methods for synthetic details). The poor PEC performance of $\mathrm{BST} / \mathrm{Bi}_{2} \mathrm{~S}_{3}$ and $\mathrm{BST} / \mathrm{MoS}_{2}$ agrees with the picture that reducing interfacial defects is important to improve charge separation (Supplementary Fig. 29).

We further quantified the $\mathrm{H}_{2}$ evolution rate during the $\mathrm{PEC} \mathrm{H}_{2}$ production reaction under NIR light. The BST-MHs achieved high performance of more than $46 \mu \mathrm{mol} \mathrm{cm}^{-3} \mathrm{~h}^{-1}$ at $0.60 \mathrm{~V}_{\mathrm{RHE}}$ under NIR light (Fig. 4a). In contrast, the $\mathrm{H}_{2}$ production rates for BST-NTs, BST-NSs, and the mixture of the two were 22, 15, and $34 \mu \mathrm{mol} \mathrm{cm}{ }^{-3} \mathrm{~h}^{-1}$, respectively. The photocurrent densities showed a slight decay after a continuous $5 \mathrm{~h}$ measurement, which was attributed to the hole-accumulation-induced photocorrosion (Fig. 4b). The $\mathrm{H}_{2}$ evolution rate of the BST-MH photoanode reached $85 \mu \mathrm{mol} \mathrm{cm} \mathrm{cm}^{-3} \mathrm{~h}^{-1}$ at $0.60 \mathrm{~V}_{\mathrm{RHE}}$ under visible light illumination $(>420 \mathrm{~nm})$ (Supplementary Fig. 30a). Meanwhile, the morphology and crystal structure showed no observable changes (Supplementary Fig. 30b, c). XPS spectra (Supplementary Fig. 30d-f) indicated that the photoanode surface was slightly oxidized after the long-term reactions, which is in accordance with the modest performance degradation.

\section{Discussion}

In summary, we have established NIR-MHs as a class of new photoanode materials, which possess a wide light-harvesting spectral range and efficient charge separation. In delving deeper, the BST-MHs constructed by few-layer nanosheets grown on nanotubes showed a high absorption of NIR light $(\lambda>800 \mathrm{~nm})$ and a high photoinduced carrier separation due to the latticematched morphological heterojunctions. The BST-MH photoanode showed an IPCE of $36 \%$ at $800 \mathrm{~nm}$ in an electrolyte solution containing hole scavengers and a photocurrent density of $1.5 \mathrm{~mA} \mathrm{~cm}^{-2}$ at $0.60 \mathrm{~V}_{\mathrm{RHE}}$ under NIR light. When we introduce CoSeTe, the BST/CoSeTe- $2 \%$ photoanode produces hydrogen under NIR light for $120 \mathrm{~h}$. This study offers new possibilities to engineer efficient NIR-active PEC devices by integrating the advantages of NBG semiconductors into a lattice-matched morphological heterojunction configuration.

\section{Methods}

Chemicals. $\mathrm{Na}_{2} \mathrm{TeO}_{3}(98 \%)$ was purchased from Maya Reagent (China). Polyvinylpyrrolidone (PVP, K30), Se powder (99\%), hydrazine hydrate (85\%, w/w\%), aqueous ammonium solution (25-28\%, w/w\%), acetone, ethylene glycol (EG) thiourea, $\mathrm{Bi}\left(\mathrm{NO}_{3}\right)_{3} \cdot 5 \mathrm{H}_{2} \mathrm{O}, \mathrm{Na}_{2} \mathrm{SeO}_{3}, \mathrm{Na}_{2} \mathrm{TeO}_{3}, \mathrm{KOH},\left(\mathrm{NH}_{4}\right)_{6} \mathrm{Mo}_{7} \mathrm{O}_{24} \cdot 4 \mathrm{H}_{2} \mathrm{O}, N, N$ dimethylformamide (DMF), trichloromethane $\left(\mathrm{CHCl}_{3}\right)$, sodium sulfide $\left(\mathrm{Na}_{2} \mathrm{~S}\right)$, and sodium sulfite $\left(\mathrm{Na}_{2} \mathrm{SO}_{3}\right)$ were purchased from the Shanghai Reagent Company (P. R. China). All chemicals were used as received without further purification.

Synthesis of Te nanowires. In a typical synthesis ${ }^{40}, 10.0 \mathrm{~g} \mathrm{PVP}, 0.922 \mathrm{~g} \mathrm{Na}_{2} \mathrm{TeO}_{3}$, and $320 \mathrm{~mL}$ double-distilled deionized water (DIW, 18.23 M ) were added into a
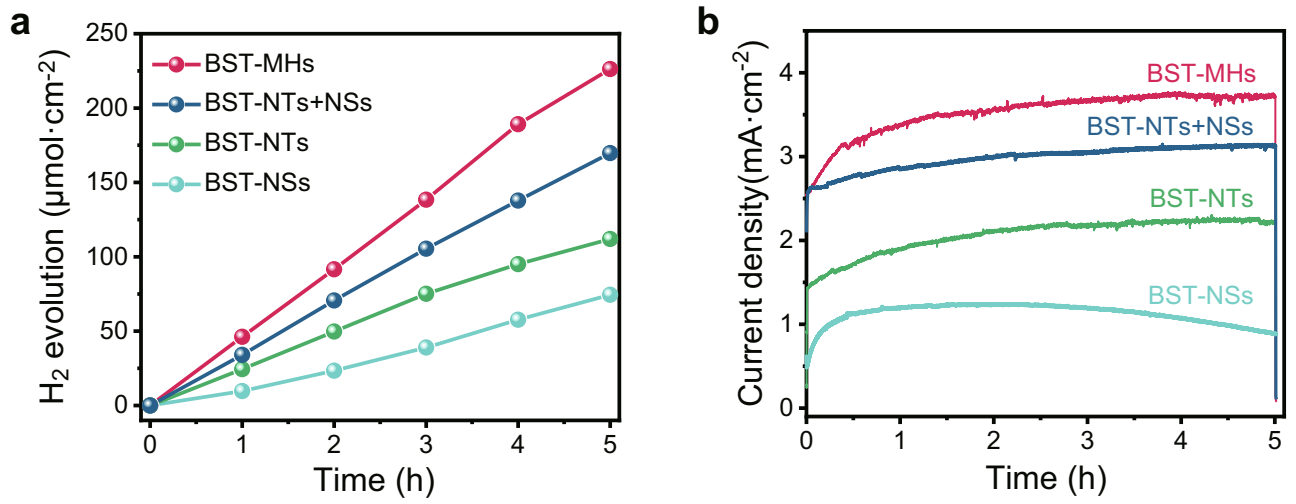

Fig. 4 PEC $\mathbf{H}_{\mathbf{2}}$ evolution. a, b $\mathrm{H}_{2}$ evolution (a) and photocurrent densities (b), of the BST-MH, BST-NT, BST-NS, and BST-NT + BST-NS photoanodes at 0.6 $V_{\mathrm{RHE}}$. The experiments were measured in an electrolyte solution containing $0.08 \mathrm{~mol} \mathrm{~L}^{-1} \mathrm{Na}_{2} \mathrm{~S}$ and $0.50 \mathrm{~mol} \mathrm{~L}^{-1} \mathrm{Na}_{2} \mathrm{SO}_{3}$ as hole scavengers ( $\mathrm{pH}=12.9$ ) under NIR light irradiation $\left(\lambda>800 \mathrm{~nm}, 100 \mathrm{~mW} \mathrm{~cm}^{-2}\right)$. 
$500 \mathrm{~mL}$ Teflon-lined stainless steel autoclave under vigorous magnetic stirring. Then, $33.5 \mathrm{~mL}$ aqueous ammonium solution and $16.5 \mathrm{~mL}$ hydrazine hydrate were added to the solution in the autoclave. After $30 \mathrm{~min}$, the autoclave was maintained at $180^{\circ} \mathrm{C}$ for $3 \mathrm{~h}$ and then allowed to cool to room temperature naturally.

Synthesis of Te $\mathbf{T e}_{\mathbf{x}} \mathrm{Se}_{\mathbf{y}} @$ Se nanowires. In a typical synthesis ${ }^{39}, \mathrm{Te}_{\mathbf{x}} \mathrm{Se}_{\mathrm{y}} @ \mathrm{Se}$ nanowires with different Te:Se molar ratios can be obtained using Te nanowires as templates and controlling the Te:Se ratio by varying the amount of Se-hydrazine solution. In order to synthesize $\mathrm{Te}_{\mathrm{x}} \mathrm{Se}_{\mathrm{y}} @ \mathrm{Se}$ nanowires with a Te:Se ratio of 1:8, $0.4 \mathrm{mmol}$ of the Te nanowire solution was mixed with $100 \mathrm{~mL}$ acetone to extract the Te nanowires. Then, the Te nanowires were redispersed in $70 \mathrm{~mL}$ DIW to form a homogenous solution. After that, a certain amount of Se-hydrazine solution (3.2 mmol Se powder dissolved in $1.0 \mathrm{~mL}$ hydrazine hydrate) was dropped slowly into the previous solution and DIW was added to maintain a total final volume of $80 \mathrm{~mL}$. The final solution was heated at $40^{\circ} \mathrm{C}$ for $12 \mathrm{~h}$ under magnetic stirring. Finally, the solution was aged at $80^{\circ} \mathrm{C}$ for another $12 \mathrm{~h}$.

\section{Synthesis of BiSeTe ternary alloy lattice-matched morphological hetero-} junctions. In order to prepare the BiSeTe ([Te]:[Se] $=1: 8)$ ternary alloy latticematched morphological heterojunctions (BST-MHs), $0.6 \mathrm{mmol}$ of $\mathrm{Bi}\left(\mathrm{NO}_{3}\right)_{3} \cdot 5 \mathrm{H}_{2} \mathrm{O}$, $38 \mathrm{~mL}$ of DIW, and $2 \mathrm{~mL}$ of hydrazine hydrate, $0.9 \mathrm{mmol}$ of $\mathrm{Te}_{\mathrm{x}} \mathrm{Se}_{\mathrm{y}} @ \mathrm{Se}$ nanowires solution ([Te]:[Se] $=1: 8$ ) were mixed in a $100 \mathrm{~mL}$ Teflon-lined stainless steel autoclave under magnetic stirring. Then the autoclave was sealed and maintained at $160^{\circ} \mathrm{C}$ for $12 \mathrm{~h}$. After the reaction, the final products were collected by centrifugation $(11,000 \mathrm{xg}, 3 \mathrm{~min})$ and washed three times with ethanol.

Synthesis of BiSeTe ternary alloy nanotubes. About $0.9 \mathrm{mmol}$ of $\mathrm{Te}_{\mathrm{x}} \mathrm{Se}_{\mathrm{y}} @ \mathrm{Se}$ nanowires ([Te]:[Se] $=1: 8)$ solution were collected by centrifugation $(17,000 \mathrm{xg}, 5$ min) to extract the $\mathrm{Te}_{\mathrm{x}} \mathrm{Se}_{\mathrm{y}} @ \mathrm{Se}$ nanowires. Then the Te $\mathrm{Se}_{\mathrm{y}} @ S e$ nanowires were redispersed in $78 \mathrm{~mL}$ EG to form a homogenous solution. After that, $0.6 \mathrm{mmol}$ of $\mathrm{Bi}\left(\mathrm{NO}_{3}\right)_{3} \cdot 5 \mathrm{H}_{2} \mathrm{O}, 2 \mathrm{~mL}$ of hydrazine hydrate, and $\mathrm{Te}_{\mathrm{x}} \mathrm{Se}_{\mathrm{y}} @ S e$ nanowire solution were mixed in a $100 \mathrm{~mL}$ Teflon-lined stainless steel autoclave under magnetic stirring. Then the autoclave was sealed and maintained at $160^{\circ} \mathrm{C}$ for $12 \mathrm{~h}$. After the reaction, the final products were collected by centrifugation $(11,000 \mathrm{xg}, 3 \mathrm{~min})$ and washed three times with ethanol.

Synthesis of BiSeTe ternary alloy nanosheets. In the synthesis of BST-NSs, $0.4 \mathrm{mmol}$ of $\mathrm{Bi}\left(\mathrm{NO}_{3}\right)_{3} \cdot 5 \mathrm{H}_{2} \mathrm{O}, 0.533 \mathrm{mmol} \mathrm{Na} \mathrm{SeO}_{3}, 0.067 \mathrm{mmol} \mathrm{Na}_{2} \mathrm{TeO}_{3}, 1.0 \mathrm{~g}$ PVP, and $1.0 \mathrm{~g} \mathrm{KOH}$ were dissolved in $80 \mathrm{~mL}$ EG and heated to $200{ }^{\circ} \mathrm{C}$ for $24 \mathrm{~h}$. After the reaction, the final products were collected by centrifugation (4200 $\mathrm{xg}, 3$ min) and washed three times with ethanol.

Synthesis of $\mathbf{B i S e T e} / \mathrm{Bi}_{\mathbf{2}} \mathbf{S}_{\mathbf{3}}$. About $0.3 \mathrm{mmol}$ of $\mathrm{Bi}\left(\mathrm{NO}_{3}\right)_{3} \cdot 5 \mathrm{H}_{2} \mathrm{O}, 40 \mathrm{~mL}$ of DIW, $30 \mathrm{~mL}$ of EG, and $0.3 \mathrm{~g}$ of thiourea, $10 \mathrm{~mL}$ of BST-NTs solution were mixed in a $100 \mathrm{~mL}$ Teflon-lined stainless steel autoclave under magnetic stirring. Then the autoclave was sealed and maintained at $110^{\circ} \mathrm{C}$ for $12 \mathrm{~h}$. After the reaction, the final products were collected by centrifugation $(11,000 \mathrm{xg}, 3 \mathrm{~min})$ and washed three times with ethanol.

Synthesis of BiSeTe/MoS $\mathbf{2}$. A total of, $20 \mathrm{~mL}$ BST-NTs solution was collected by centrifugation $(11,000 \mathrm{xg}, 5 \mathrm{~min})$ to extract the BST-NTs. Then the BST-NTs were re-dispersed in $80 \mathrm{~mL}$ DIW to form a homogenous solution. After that, $0.15 \mathrm{mmol}$ of $\left(\mathrm{NH}_{4}\right)_{6} \mathrm{Mo}_{7} \mathrm{O}_{24} \cdot 4 \mathrm{H}_{2} \mathrm{O}$ and $4.5 \mathrm{mmol}$ of thiourea were mixed in a $100 \mathrm{~mL}$ Teflonlined stainless steel autoclave under magnetic stirring. Then the autoclave was sealed and maintained at $180{ }^{\circ} \mathrm{C}$ for $12 \mathrm{~h}$. After the reaction, the final products were collected by centrifugation $(11,000 \mathrm{xg}, 3 \mathrm{~min})$ and washed three times with ethanol.

Synthesis of BiSeTe/CoSeTe. We chose BiSeTe/CoSeTe-1\% to demonstrate the synthesis. About $0.594 \mathrm{mmol}$ of $\mathrm{Bi}\left(\mathrm{NO}_{3}\right)_{3} \cdot 5 \mathrm{H}_{2} \mathrm{O}, 0.009 \mathrm{mmol}$ of $\mathrm{Co}\left(\mathrm{NO}_{3}\right)_{2} \cdot 6 \mathrm{H}_{2} \mathrm{O}$, $38 \mathrm{~mL}$ of DIW, $2 \mathrm{~mL}$ of hydrazine hydrate, and $0.9 \mathrm{mmol}$ of $\mathrm{Te}_{\mathrm{x}} \mathrm{Se}_{\mathrm{y}} @ \mathrm{Se}$ nanowires solution ([Te]:[Se] $=1: 8$ ) were mixed in a $100 \mathrm{~mL}$ Teflon-lined stainless steel autoclave under magnetic stirring. The autoclave was sealed and maintained at $160{ }^{\circ} \mathrm{C}$ for $12 \mathrm{~h}$. After the reaction, the final products were collected by centrifugation $(11,000 \mathrm{xg}, 3 \mathrm{~min})$ and washed three times with ethanol.

Photoanode fabrication. The photoanodes were fabricated by a Langmuir-Blodgett technology (Nima Technology, 312D) with DIW as a subphase $\mathrm{e}^{50}$. The prepared BSTMH solution $(1 \mathrm{~mL})$ was centrifuged after adding alcohol $(3 \mathrm{~mL})$ and then the BSTMHs were re-dispersed into a stirred solution of chloroform $(1 \mathrm{~mL})$ and DMF $(1 \mathrm{~mL})$ to form a homogeneous solution. The final solution was dispensed from a $100 \mu \mathrm{L}$ syringe onto the water subphase dropwise to form a film. After $10 \mathrm{~min}$, the film was compressed by a Wilhelmy plate with a compression rate of $20 \mathrm{~cm}^{2} \mathrm{~min}^{-1}$. The final film was lifted with an FTO glass $(1 \mathrm{~cm} \times 3 \mathrm{~cm})$. By repeating the lifting process, the thickness of BST-MH film on the FTO glass can be modulated.

Femtosecond transient absorption (TA) measurement. We used BST-MH, BST-NT, and BST-NS films for TA measurement. For the TA measurement (Time-
Tech Spectra, TA100), the fundamental output from Yb:KGW laser (1030 nm, $100 \mathrm{kHz}, 220 \mathrm{fs}$ Gaussian fit, Light Conversion Ltd) was separated into two light beams. One beam was introduced to the optical parametric amplifier (ORPHEUS $\mathrm{N}$, Light Conversion Ltd) to produce a $460 \mathrm{~nm}$ pump beam, and the other was focused onto a YAG plate to produce a white light continuum probe beam. A linear array detector was used to collect the transmitted probe light from the sample.

Photoelectrochemical current measurement. The $\mathrm{PEC} \mathrm{H}_{2}$ production performance of the photoanodes was tested on a CHI760D electrochemistry workstation in a typical three-electrode configuration. The counter electrode was a Pt foil, and the reference electrode was an $\mathrm{Ag} / \mathrm{AgCl}$ electrode. The simulated solar illumination was supplied by a $300 \mathrm{~W}$ xenon lamp equipped with a NIR filter $(\lambda>800 \mathrm{~nm})$ or a UV light filter $(\lambda>420 \mathrm{~nm})$. The intensity of the incident light was calibrated to $100 \mathrm{~mW} \mathrm{~cm}^{-2}$ with a power meter. Photocurrent measurements were measured in an electrolyte solution containing $0.08 \mathrm{~mol} \mathrm{~L}^{-1} \mathrm{Na}_{2} \mathrm{~S}$ and $0.50 \mathrm{~mol} \mathrm{~L}^{-1} \mathrm{Na}_{2} \mathrm{SO}_{3}$ as hole scavengers $(\mathrm{pH}=12.9)$. The electrolyte was thoroughly deaerated by purging with nitrogen before measurements. The photocurrents were collected at a range of -0.9 to $-0.15 \mathrm{~V}$ vs $\mathrm{Ag} / \mathrm{AgCl}$ with a scan rate of $10 \mathrm{mV} \mathrm{s}^{-1}$. In order to transform the potential vs $\mathrm{Ag} / \mathrm{AgCl}$ to reversible hydrogen electrode (RHE), the following equation was used ${ }^{51,52}$

$$
E_{\mathrm{RHE}}=E_{\mathrm{Ag} / \mathrm{AgCl}}+0.059 \mathrm{pH}+E_{\mathrm{Ag} / \mathrm{AgCl}}^{\circ}
$$

The $E_{\mathrm{Ag} / \mathrm{AgCl}}^{\circ}(3 \mathrm{M} \mathrm{KCl})=0.197$ at $25^{\circ} \mathrm{C}$.

Photoelectrochemical $\mathbf{H}_{\mathbf{2}}$ evolution measurement. Photoelectrochemical $\mathrm{H}_{2}$ production was measured in a PEC cell system connected to a glass-closed gas circulation system. For each measurement, the BST-MH photoanode was immersed in an electrolyte $(200 \mathrm{~mL})$ containing $\mathrm{Na}_{2} \mathrm{SO}_{3}(0.5 \mathrm{M})$ and $\mathrm{Na}_{2} \mathrm{~S}(0.08 \mathrm{M})$ as hole scavengers. The electrolyte was evacuated three times to completely remove air. The test was conducted under a $300 \mathrm{~W}$ xenon lamp equipped with a NIR filter $\left(\lambda>800 \mathrm{~nm}, 100 \mathrm{~mW} \mathrm{~cm}^{-2}\right)$ or a UV light filter $\left(\lambda>420 \mathrm{~nm}, 100 \mathrm{~mW} \mathrm{~cm}^{-2}\right)$. Using Ar as a carrier gas, the $\mathrm{H}_{2}$ evolved from the PEC cell system was quantified through a gas chromatograph (Agilent Technologies Corporation) equipped with a thermal conductive detector. The gas products were sampled every hour.

Mott-Schottky Plot. The Mott-Schottky Plot was collected with a CHI760D electrochemistry workstation through an impedance-capacitance scan technique with an amplitude of $10 \mathrm{mV}$ and different frequencies $(0.5,1$, and 1.5$)$. The electrolyte and the photoanodes were the same as that in PEC current measurements. The donor concentration can be calculated by the equation: ${ }^{52}$

$$
\frac{1}{C^{2}}=\frac{2\left(V-V_{\mathrm{f}}-k T / e\right)}{e \varepsilon \varepsilon_{0} N_{\mathrm{D}} A^{2}}
$$

where $C$ is the capacitance of the photoanode, $e$ is the charge of an electron $(1.6 \times$ $\left.10^{-19} \mathrm{C}\right), \varepsilon$ is the dielectric constant of $\mathrm{Bi}_{2} \mathrm{Se}_{3}{ }^{38}, \varepsilon_{o}$ is the vacuum permittivity (8. $854187817 \times 10^{-12} \mathrm{~F} \mathrm{~m}^{-1}$ ), $V$ is the applied bias (vs RHE), $V_{\mathrm{f}}$ is the flat-band potential (vs RHE), $k$ is the Boltzmann constant, $N_{\mathrm{D}}$ is the donor density $\left(\mathrm{cm}^{-3}\right), A$ is the surface area of the photoanodes, and $T$ is the temperature $(\mathrm{K})$.

IPCE measurement. IPCE was also measured on the CHI760D electrochemistry workstation and in the same electrolyte using a three-electrode configuration at 0.6 $\mathrm{V}_{\mathrm{RHE}}$, with solar irradiation from 400 to $1200 \mathrm{~nm}$. The IPCE can be calculated by the equation: ${ }^{52}$

$$
\operatorname{IPCE}(\%)=\frac{1240 \times J}{\lambda \times P} \times 100
$$

Where the $J$ is the photocurrent density $\left(\mathrm{mA} \mathrm{cm}^{-2}\right), P$ is the light power density $\left(\mathrm{mW} \mathrm{cm}^{-2}\right)$ at $\lambda$, and $\lambda$ is the wavelength of incident light (nm).

Characterization. The morphology of the nanostructures was investigated by TEM using a Hitachi H-7700 microscope at $100 \mathrm{kV}$. SEM images were obtained with a field emission scanning electron microanalyzer (Zeiss Supra 40) at an acceleration voltage of $5 \mathrm{kV}$. XRD patterns were measured on a Rigaku DMax $-\gamma$ A rotation anode $\mathrm{X}$-ray diffractometer equipped with graphite monochromatized $\mathrm{Cu}-\mathrm{K}$ radiation $(\lambda=1.54178 \AA)$. HRTEM, high-angle annular dark-field scanning transmission electron microscopy (HAADF-STEM) image, and energy dispersive $\mathrm{X}$-ray (EDX) elemental mapping were obtained by JEOL $2100 \mathrm{~F}$, FEI Tecnai G2 F20 S-Twin microscope, and Talos F200X at $200 \mathrm{kV}$. X-ray photoelectron spectroscopy (XPS) was recorded using an ESCALAB-MKII x-ray photoelectron spectrometer equipped with $\mathrm{Mg}$ Ka radiation as an exciting source. Raman spectra were detected by a Renishaw System 2000 spectrometer using the $514.5 \mathrm{~nm}$ line of $\mathrm{Ar}^{+}$for excitation. UV-vis-NIR absorption spectra were obtained by a Shimadzu UV3600 spectrometer at room temperature. AFM was performed by a Bruker Dimension Icon. The infrared spectra (IR) was carried out on a NICOLET Fourier transform infrared spectrometer using pressed $\mathrm{KBr}$ pellets. Thermal gravimetric analysis (TGA) of the samples was obtained on a Shimadzu TA-50 thermal analyzer (Shimadzu) at a heating rate of $10^{\circ} \mathrm{C} \mathrm{min}-1$ from room temperature to $600^{\circ} \mathrm{C}$. The thickness of the BST-MHs film was measured on a Bruker DektakXT. 
The microtomy was finished on Leica Ultra CUT UC7. In addition, UPS was performed at the Catalysis and Surface Science Endstation (BL11U beamline) in the National Synchrotron Radiation Laboratory (NSRL) in Hefei, China. A sample bias of $-5 \mathrm{~V}$ was applied to observe the secondary electron cutoff with a photon energy of $40.0 \mathrm{eV}$. The work function is determined by the difference between the photon energy and the binding energy of the secondary cutoff edge. In actual testing, the $\mathrm{Au}$ layer is assigned to $0 \mathrm{eV}$ and acts as a reference for $E_{\mathrm{f}}$. EIS was measured with a CHI760D electrochemical workstation using a three-electrode configuration.

\section{Data availability}

The data that support the findings of this study are available on request from the corresponding authors (M.-R.G., S.-H.Y., or E.H.S.)

Received: 23 February 2021; Accepted: 17 June 2021;

Published online: 14 July 2021

\section{References}

1. Turner, J. A. A realizable renewable energy future. Science $\mathbf{2 8 5}, 687-689$ (1999).

2. Turner, J. A. Sustainable hydrogen production. Science 305, 972-974 (2004).

3. Schultz, D. M. \& Yoon, T. P. Solar synthesis: prospects in visible light photocatalysis. Science 343, 1239176 (2014).

4. Tachibana, Y., Vayssieres, L. \& Durrant, J. R. Artificial photosynthesis for solar water-splitting. Nat. Photon. 6, 511-518 (2012).

5. Wang, Q. \& Domen, K. Particulate photocatalysts for light-driven water splitting: mechanisms, challenges, and design strategies. Chem. Rev. 120, 919-985 (2020).

6. Luo, D. Enhanced photovoltage for inverted planar heterojunction perovskite solar cells. Science 360, 1442-1446 (2018).

7. Laskowski, F. A. L. et al. Nanoscale semiconductor/catalyst interfaces in photoelectrochemistry. Nat. Mater. 19, 69-77 (2020).

8. Fujishima, A. \& Honda, K. Electrochemical photolysis of water at a semiconductor electrode. Nature 238, 37-38 (1972).

9. Steven, Y. \& Reece, D. G. N. Wireless solar water splitting using silicon-based semiconductors and Earth-abundant catalysts. Science 334, 645-648 (2011).

10. Li, Y. et al. Pulsed axial epitaxy of colloidal quantum dots in nanowires enables facet-selective passivation. Nat. Commun. 9, 4947 (2018).

11. Yu, Y. et al. Enhanced photoelectrochemical efficiency and stability using a conformal $\mathrm{TiO}_{2}$ film on a black silicon photoanode. Nat. Energy 2, 17045 (2017).

12. Kuang, Y., Yamada, T. \& Domen, K. Surface and interface engineering for photoelectrochemical water oxidation. Joule 1, 1-16 (2017).

13. Hisatomi, T. et al. Recent advances in semiconductors for photocatalytic and photoelectrochemical water splitting. Chem. Soc. Rev. 43, 7520-7535 (2014).

14. Li, Z. et al. Photoelectrochemical cells for solar hydrogen production: current state of promising photoelectrodes, methods to improve their properties, and outlook. Energy Environ. Sci. 6, 347-370 (2013).

15. Nellist, M. R. et al. Semiconductor-electrocatalyst interfaces: theory, experiment, and applications in photoelectrochemical water splitting. Acc. Chem. Res. 49, 733-740 (2016).

16. Wang, D., Sheng, T., Chen, J., Wang, H. F. \& Hu, P. Identifying the key obstacle in photocatalytic oxygen evolution on rutile $\mathrm{TiO}_{2}$. Nat. Catal. 1 , 291-299 (2018).

17. Zhuang, T. T. et al. Integration of semiconducting sulfides for full-spectrum solar energy absorption and efficient charge separation. Angew. Chem. Int. Ed. 128, 6506-6510 (2016).

18. Wang, D. Y. et al. Iron pyrite/titanium dioxide photoanode for extended near infrared light harvesting in a photoelectrochemical cell. Sci. Rep. 6, 20397 (2016).

19. Li, K. et al. Hexagonal@Cubic CdS Core@Shell nanorod photocatalyst for highly active production of $\mathrm{H}_{2}$ with unprecedented stability. Adv. Mater. 28, 8906-8911 (2016).

20. Kim, T. W. \& Choi, K.-S. Nanoporous $\mathrm{BiVO}_{4}$ photoanodes with dual-layer oxygen evolution catalysts for solar water splitting. Science 343, 990-994 (2014).

21. Kim, T. W., Ping, Y., Galli, G. A. \& Choi, K. S. Simultaneous enhancements in photon absorption and charge transport of bismuth vanadate photoanodes for solar water splitting. Nat. Commun. 6, 8769 (2015).

22. Shockley, W. \& Queisser, H. J. Detailed balance limit of efficiency of $\mathrm{p}-\mathrm{n}$ junction solar cells. J. Appl. Phys. 32, 510-519 (1961)

23. Wang, D., Zhu, L., Chen, J.-F. \& Dai, L. Liquid marbles based on magnetic upconversion nanoparticles as magnetically and optically responsive miniature reactors for photocatalysis and photodynamic therapy. Angew. Chem. Int. Ed. 55, 10795-10799 (2016).
24. Jia, $\mathrm{H}$. et al. Near-infrared light-induced photocurrent from a $\left(\mathrm{NaYF}_{4}: \mathrm{Yb}-\right.$ $\mathrm{Tm}) /\left(\mathrm{Cu}_{2} \mathrm{O}\right)$ composite thin film. Adv. Energy Mater. 5, 1401041 (2015).

25. Chen, Y. C. et al. Au@Nb@ $\mathrm{H}_{\mathrm{x}} \mathrm{K}_{1-\mathrm{x}} \mathrm{NbO}_{3}$ nanopeapods with near-infrared active plasmonic hot-electron injection for water splitting. Nat. Commun. 9, 232 (2018).

26. Tian, J. et al. A $\mathrm{Bi}_{2} \mathrm{WO}_{6}$-based hybrid photocatalyst with broad spectrum photocatalytic properties under UV, visible, and near-infrared irradiation. Adv. Mater. 25, 5075-5080 (2013).

27. Sang, Y. et al. From UV to near-infrared, $\mathrm{WS}_{2}$ nanosheet: a novel photocatalyst for full solar light spectrum photodegradation. Adv. Mater. 27, 363-369 (2015).

28. Brongersma, M. L., Halas, N. J. \& Nordlander, P. Plasmon-induced hot carrier science and technology. Nat. Nanotechnol. 10, 25-34 (2015).

29. Wei, D. et al. Band gap engineering of $\mathrm{In}_{2} \mathrm{TiO}_{5}$ for $\mathrm{H}_{2}$ production under nearinfrared light. ACS Appl. Mater. Interfaces 7, 20761-20768 (2015).

30. Zhou, Y. et al. ltrasmall nanoplatelets: the ultimate tuning of optoelectronic properties. Adv. Energy Mater. 7, 1602728 (2017)

31. Xi, G. et al. Ultrathin $\mathrm{W}_{18} \mathrm{O}_{49}$ nanowires with diameters below $1 \mathrm{~nm}$ : synthesis, near-infrared absorption, photoluminescence, and photochemical reduction of carbon dioxide. Angew. Chem. Int. Ed. 51, 2395-2399 (2012).

32. Abdi, F. F. et al. Efficient solar water splitting by enhanced charge separation in a bismuth vanadate-silicon tandem photoelectrode. Nat. Commun. $\mathbf{4}$ 3195-3197 (2013)

33. Bai, Y. et al. Homophase junction for promoting spatial charge separation in photocatalytic water splitting. ACS Catal. 9, 3242-3252 (2019)

34. Sun, Y. et al. Atomically thick bismuth selenide freestanding single layers achieving enhanced thermoelectric energy harvesting. J. Am. Chem. Soc. 134, 20294-20297 (2012).

35. Zhang, H., Zhang, X., Liu, C., Lee, S. T. \& Jie, J. High-responsivity, highdetectivity, ultrafast topological insulator $\mathrm{Bi}_{2} \mathrm{Se}_{3} / \mathrm{Silicon}$ heterostructure broadband photodetectors. ACS Nano 10, 5113-5122 (2016).

36. Song, G. et al. Perfluorocarbon-loaded hollow $\mathrm{Bi}_{2} \mathrm{Se}_{3}$ nanoparticles for timely supply of oxygen under near-infrared light to enhance the radiotherapy of cancer. Adv. Mater. 28, 2716-2723 (2016).

37. Wang, B., Niu, X., Ouyang, Y., Zhou, Q. \& Wang, J. Ultrathin semiconducting $\mathrm{Bi}_{2} \mathrm{Te}_{2} \mathrm{~S}$ and $\mathrm{Bi}_{2} \mathrm{Te}_{2} \mathrm{Se}$ with high electron mobilities. J. Phys. Chem. Lett. 9 , 487-490 (2018).

38. Ahmed, R. et al. Synthesis and material properties of $\mathrm{Bi}_{2} \mathrm{Se}_{3}$ nanostructures deposited by SILAR. J. Phys. Chem. C. 122, 12052-12060 (2018).

39. Yang, Y. et al. A new generation of alloyed multimetal chalcogenide nanowires by chemical transformation. Sci. Adv. 1, e1500714 (2015).

40. Qian, H. S. et al. High-quality luminescent tellurium nanowires of several nanometers in diameter and high aspect ratio synthesized by a poly (vinyl pyrrolidone)-assisted hydrothermal process. Langmuir 22, 3830-3835 (2006).

41. Vargas, A. et al. The changing colors of a quantum-confined topological insulator. ACS Nano 9, 1222-1230 (2014).

42. Sun, L. et al. Preparation of few-layer bismuth selenide by liquid-phaseexfoliation and its optical absorption properties. Sci. Rep. 4, 4794 (2014)

43. Crowley, J. M., Tahir-Kheli, J. \& Goddard, W. A. 3rd Accurate ab initio quantum mechanics simulations of $\mathrm{Bi}_{2} \mathrm{Se}_{3}$ and $\mathrm{Bi}_{2} \mathrm{Te}_{3}$ topological insulator surfaces. J. Phys. Chem. Lett. 6, 3792-3796 (2015).

44. Kong, D. et al. Rapid surface oxidation as a source of surface degradation factor for $\mathrm{Bi}_{2} \mathrm{Se}_{3}$. ACS Nano 5, 4689-4703 (2011).

45. Jiang, Y., Zhu, Y.-J. \& Cheng, G. F. Synthesis of $\mathrm{Bi}_{2} \mathrm{Se}_{3}$ nanosheets by microwave heating using an ionic liquid. Cryst. Growth Des. 6, 2174-2176 (2006)

46. Liu, J.-W. et al. Mesostructured assemblies of ultrathin superlong tellurium nanowires and their photoconductivity. J. Am. Chem. Soc. 132, 8945-8952 (2010).

47. Maiolo, J. R. et al. Macroporous silicon as a model for silicon wire array solar cells. J. Phys. Chem. C. 112, 6194-6201 (2008).

48. Tsuji, I., Kato, H., Kobayash, H. \& Kudo, A. Photocatalytic $\mathrm{H}_{2}$ evolution reaction from aqueous solutions over band structure-controlled $(\mathrm{AgIn})_{\mathrm{x}} \mathrm{Zn}_{2(1-\mathrm{x})} \mathrm{S}_{2}$ solid solution photocatalysts with visible-light response and their surface nanostructures. J. Am. Chem. Soc. 126, 13406-13413 (2004).

49. Kornienko, N. et al. Growth and photoelectrochemical energy conversion of wurtzite indium phosphide nanowire arrays. ACS Nano 10, 5525-5535 (2016).

50. Liu, J. W., Wang, J. L., Wang, Z. H., Huang, W. R. \& Yu, S. H. Manipulating nanowire assembly for flexible transparent electrodes. Angew. Chem. Int. Ed. 53, 13477-13482 (2014).

51. Dos, Santos et al. A hole inversion layer at the $\mathrm{BiVO}_{4} / \mathrm{Bi}_{4} \mathrm{~V}_{2} \mathrm{O}_{11}$ interface produces a high tunable photovoltage for water splitting. Sci. Rep. 6, 31406 (2016).

52. Kim, J. H. et al. Wireless solar water splitting device with robust cobaltcatalyzed, dual-doped $\mathrm{BiVO}_{4}$ photoanode and perovskite solar cell in tandemA dual absorber artificial leaf. ACS Nano 9, 11820-11829 (2015).

\section{Acknowledgements}

This work was supported by the National Natural Science Foundation of China (Grants 51732011, 21431006, U1932213, 81788101, and 11227901), the Foundation for 
Innovative Research Groups of the National Natural Science Foundation of China (Grant 21521001), Key Research Program of Frontier Sciences, CAS (Grant QYZDJ-SSWSLH036), the Users with Excellence and Scientific Research Grant of Hefei Science Center of CAS (2015HSC-UE007), China Postdoctoral Science Foundation (BH2060000143, BH2060000155), the Fundamental Research Funds for the Central Universities (WK2060000031). This work was partially carried out at the USTC Center for Micro and Nanoscale Research and Fabrication.

\section{Author contributions}

S.-H.Y., M.-R.G., and E.H.S. supervised the project and wrote the paper. G.-Q.L., Y.Y., Y.L., and T.T.Z. conceived the idea and wrote the paper. G.-Q.L. carried out the experiments, analyzed the results, and wrote the paper. J.W. edited the manuscript. H.-X.J. and J.Z. performed XPS and UPS measurements. X.-F.L. and H.M.Z. provided TA measurements and helpful discussion. J.T. conducted HRTEM measurements. J.-L.P. provided a photoelectric test. L.W. analyzed the crystal structure and growth mechanism. L.-A.S. performed SEM measurements. Y.L. and Y.-X.P. assisted with the photocatalysis experiments. All authors discussed the results and assisted during manuscript preparation.

\section{Competing interests}

The authors declare no competing interests.

\section{Additional information}

Supplementary information The online version contains supplementary material available at https://doi.org/10.1038/s41467-021-24569-9.
Correspondence and requests for materials should be addressed to M.-R.G., S.-H.Y. or E.H.S.

Peer review information Nature Communications thanks Ho Won Jang and the other, anonymous, reviewer(s) for their contribution to the peer review of this work.

Reprints and permission information is available at http://www.nature.com/reprints

Publisher's note Springer Nature remains neutral with regard to jurisdictional claims in published maps and institutional affiliations.

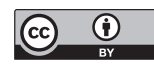

Open Access This article is licensed under a Creative Commons Attribution 4.0 International License, which permits use, sharing, adaptation, distribution and reproduction in any medium or format, as long as you give appropriate credit to the original author(s) and the source, provide a link to the Creative Commons license, and indicate if changes were made. The images or other third party material in this article are included in the article's Creative Commons license, unless indicated otherwise in a credit line to the material. If material is not included in the article's Creative Commons license and your intended use is not permitted by statutory regulation or exceeds the permitted use, you will need to obtain permission directly from the copyright holder. To view a copy of this license, visit http://creativecommons.org/ licenses/by/4.0/.

(C) The Author(s) 2021 